\title{
Planar and Nonplanar Solitons in Multi-Ion Dusty Plasma
}

\author{
F. Deeba ${ }^{1}$, Kazi Asraful Islam ${ }^{2 *}$, A. A. Mamun ${ }^{3}$ \\ ${ }^{1}$ Complex System Group, School of Physics, The University of Sydney, AUSTRALIA \\ ${ }^{2}$ Department of Textile \& Engineering, Sonargaon University, Banani, Dhaka, BANGLADESH \\ ${ }^{3}$ Department of Physics, Jahangirnagar University, Savar, Dhaka, BANGLADESH \\ ${ }^{*}$ Corresponding Contact: \\ Email: kazinooman@gmail.com
}

\begin{abstract}
A rigorous theoretical investigation has been made on nonlinear dust-ionacoustic (DIA) solitary waves (SWs) in a multi-ion dusty plasma system, consisting of inertial positive and negative ions, and arbitrary charged stationary dust. The dust particles have been considered as arbitrarily (either positively or negatively) charged in order to observe the effects of dust polarity on the DIA SWs. Three different approaches (K-dV (Korteweg-de Vries), mK-dV (mixed K-dV), and Gardner) have been employed to analysis the entire regime. The reductive perturbation method has been employed in all these three approaches. Using reductive perturbation method, we first derive $\mathrm{K}-\mathrm{dV}$ equation which let us to analyze both types (bright and dark) of solitons, but, in a very limited region. After that mK-dV equation has been derived which let us analyze bright soliton for a large region, but cannot show the dark soliton. Finally, we have derived the Gardner equation employing the same method, through which we were able to analyze both the bright and dark solitons for a large region. We also employed the modified Gardner (mG) equation to observe the effects of nonplanar geometry as well as time evolution of SWs. It has been found that both the positive and negative solitons depend on the ion number density of the ions, dust polarity, temperature, as well as time scale. It is also shown that the properties of the nonplanar (cylindrical and spherical) DIA-GSs are significantly different from those of the one dimensional planar ones.
\end{abstract}

Key words:

Dusty Plasma, Planar and nonplanar soliton, K-dV, mK-dV, Gardner equation, double layers, Soliton, Solitary Wave, Dust-Ion-Acoustic Wave, Multi-ion, Space Plasma 


\section{INTRODUCTION}

Now a days the nonlinear structures (viz., solitary waves, shock structure, and double layers) associated with the DIA waves have received a great deal of interest. A number of investigations have been made on these nonlinear structures (Fortov et al., 2005; Ishihara, 2007; Shukla and Eliasson, 2009; Morfill and Ivlev, 2009; Lonngren, 1983; Nakamura and Sharma, 2001; Nakamura et al., 1999; Luo et al., 1999; Charles, 2007), particularly DIA solitary waves (SWs) (Nakamura and Sharma, 2001; Bharuthram and Shukla, 1992; Popel and Yu, 1995; Mamun and Shukla, 2002; Shukla and Mamun, 2003; Popel et al., 2003), shock waves (Nakamura et al., 1999; Luo et al., 1999; Shukla, 2000; Popel et al., 2001; Mamun and Shukla, 2002; Mamun et al., 2009; Duha and Mamun, 2009), and double layers (DLs) (Mamun et al., 2009; Alinejad and Mamun, 2010; Ghosh and Bharuthram, 2008; Roychoudhury and Chatterjee, 1999). But most of these investigations (Nakamura and Sharma, 2001; Nakamura et al., 1999; Luo et al., 1999; Bharuthram and Shukla, 1992; Popel and Yu, 1995; Mamun and Shukla, 2002; Shukla and Mamun, 2003; Popel et al., 2003; Shukla, 2000; Popel et al., 2001; Mamun and Shukla, 2002; Mamun et al., 2009; Duha and Mamun, 2009; Mamun et al., 2009; Alinejad and Mamun, 2010; Ghosh and Bharuthram, 2008; Roychoudhury and Chatterjee, 1999) are concerned with single ion plasma species. However, the presence of the negative ions, which occur in both space (Sauer et al., 1994; Sauer et al., 1996; Sauer et al., 2003) and laboratory plasmas (Jacquinot, et al., 1977; Weingarten et al., 2001; Watanabe et al., 1978; Nakamura et al., 1997), significantly modify the properties of the linear (Lonngren, 1983) and nonlinear ion-acoustic waves in a plasma system (Lonngren, 1983; Nakamura, 1982). Therefore, the properties of ion-acoustic solitary waves in a dust-free plasma with negative ions have been investigated both theoretically and experimentally by many authors (Das and Tagare, 1975; Watanabe, 1984; Tajiri and Tuda, 1985; Ludwig et al., 1984; Nakamura and Tsukabayashi, 1984; Cooney et al., 1991). Later, the existence of ion-acoustic shocks has also found (Luo et al., 1998). It has been observed that the collective interactions in positive ion-negative ion plasmas (Kim and Merlino, 2007) have potential applications in natural and technological environments including the D-region of the Earths ionosphere, the Earths mesosphere, the solar photosphere, and the microelectronics plasma processing reactors. However, dusty multiion plasma has also devoted significant attention because of its vital role in understanding different types of collective processes in space environments (Shukla, 2001; Mendis and Rosenberg, 1994; Tsytovich et al., 2002) as well as in laboratory devices (Fortov et al., 2005; Merlino et al., 1998; Homann et al., 1997; Morfill et al., 2003). The presence of a fraction of negative ions in a dusty plasma changes the plasma composition and plasma transport properties (Klumov et al., 2003), as well as the dust charges (Mamun and Shukla, 2003; Kim and Merlino, 2006; Merlino and Kim, 2006). Some works have been also done on DIA solitary waves (Sayed et al., 2008; Verheest et al., 2008) and shock waves (Mamun et al., 2009; Duha, 2009) in dusty multi-ion plasma. However, all of these works (Sayed et al., 2008; Verheest et al., 2008; Mamun et al., 2009; Duha, 2009) are limited to planar geometry and too solitary and shock structures. Since the waves observed in laboratory devices are certainly not bounded to one-dimension, the investigations made on 1D (planar) nonlinear DIA waves, may not be appropriate for realistic space or laboratory dusty plasma situations. Moreover, in all of these investigations (Sayed et al., 2008; Verheest et al., 2008; Mamun et al., 2009; Duha, 2009), authors have used the K-dV or Burgers equations, which are not valid for a parametric regime corresponding to $\mathrm{A}=0$ or $\mathrm{A} \sim 0$ (where $\mathrm{A}$ is the coefficient of the nonlinear term of the K-dV or Burger equation (Mamun and Shukla, 2002; Mamun and Shukla, 2009a; Mamun and Shukla, 2009b), and A 0 means here that A is not equal 0, but 
$\mathrm{A}$ is around 0 ). This is because, the latter gives rise to infinitely large amplitude structures which break down the validity of the reductive perturbation method (Washimi and Taniuti, 1966). To the best of our knowledge, no attempt has been made on the entire regime of the planar DIA SWs as well as nonplanar DIA SWs in multi-ion plasma system. Therefore, in our present work we have employed three different approaches e.g. K-dV (Korteweg-de Vries), mK-dV (mixed K-dV), and Gardner (Deeba et al., 2012) to analysis the entire regime. In all these three approaches, the reductive perturbation method has been employed. At first, we derive $\mathrm{K}-\mathrm{dV}$ equation which let us to analyze both types (bright and dark) of solitons, but, in a very limited region. After that $\mathrm{mK}-\mathrm{dV}$ equation has been derived which let us analyze bright soliton for a large region, but cannot show the dark soliton. Then, finally we derive the Gardner equation, through which we analyze both the bright and dark solitons for a large region. We also employed the modified Gardner ( $\mathrm{mG}$ ) equation (Deeba and Mamun, 2011; Mamun and Deeba, 2012) to observe the effects of nonplanar geometry as well as time evolution on SWs.

\section{GOVERNING EqUATIONS}

In our present work, the nonlinear propagation of the DIA waves in an unmagnetized multiion dusty plasma system (consisting of inertial positive and negative ions, and arbitrary charged stationary dust) has been considered. At equilibrium, we have $Z_{\mathrm{p}} n_{\mathrm{p} 0}=Z_{\mathrm{n}} n_{\mathrm{n} 0}+1 Z_{\mathrm{d}} \mathrm{d} 0$, where $\mathrm{n}_{\mathrm{p} 0}\left(\mathrm{n}_{\mathrm{n} 0}\right) \mathrm{n}_{\mathrm{d} 0}$ is the positive ion (negative ion) dust number density at equilibrium, $Z_{\mathrm{p}}$ $\left(Z_{n}\right) Z_{d}$ is the number of charged particles residing onto the unit grain surface of positive ion (negative ion) dust, and 1 is the polarity of the dust particles. When $1=1$, the dust particles are negatively charged and when $1=-1$, the dust particles are positively charged. The dynamics of such low velocity, nonplanar DIA waves are governed by

$$
\begin{aligned}
& \partial \mathrm{n}_{\mathrm{p}} / \partial \mathrm{t}+1 / \mathrm{r}^{v} \partial / \partial \mathrm{r}\left(\mathrm{r}^{v} n_{\mathrm{p}} u_{\mathrm{p}}\right)=0, \\
& \partial \mathrm{n}_{\mathrm{n}} / \partial \mathrm{t}+1 / \mathrm{r}^{v} \partial / \partial \mathrm{r}\left(\mathrm{r}^{v} n_{n} u_{\mathrm{n}}\right)=0, \\
& \mathrm{n}_{\mathrm{p}} \partial \mathrm{u}_{\mathrm{p}} / \partial \mathrm{t}+\mathrm{n}_{\mathrm{p}} \mathrm{u}_{\mathrm{p}} \partial \mathrm{u}_{\mathrm{p}} / \partial \mathrm{r}+\mathrm{n}_{\mathrm{p}} \partial \phi / \partial \mathrm{r}+\partial \mathrm{n}_{\mathrm{p}} / \partial \mathrm{r}=0, \\
& \mathrm{n}_{\mathrm{n}} \mu \partial \mathrm{u}_{\mathrm{n}} / \partial \mathrm{t}+\mathrm{n}_{\mathrm{n}} \mathrm{u}_{\mathrm{n}} \mu \partial \mathrm{u}_{\mathrm{n}} / \partial \mathrm{r}-\mathrm{n}_{\mathrm{n}} \beta \partial \phi / \partial \mathrm{r}+\sigma \partial \mathrm{n}_{\mathrm{n}} / \partial \mathrm{r}=0, \\
& 1 / \mathrm{r}^{v} \partial / \partial \mathrm{r}\left(\mathrm{r}^{v} \partial \phi / \partial \mathrm{r}\right)=-\rho, \\
& \rho=\left[\mathrm{n}_{\mathrm{p}}-(1-\alpha) \mathrm{n}_{\mathrm{n}}-\mathrm{l} \alpha\right],
\end{aligned}
$$

where $v$ is the co-efficient of geometry. $v=0$ represents an 1D (one dimensional) planar geometry, while $v=1$ (2) represents a nonplanar cylindrical (spherical) geometry. $n_{p}\left(n_{n}\right)$ is the number density of positive (negative) ion normalized by $n_{p 0}\left(n_{n 0}\right) ; u_{p}\left(u_{n}\right)$ is the fluid speed positive (negative) ion normalized by $\mathrm{C}_{\mathrm{p}} ; \phi$ is the electrostatic wave potential normalized by $\left(T_{p} / Z_{p} e\right) ; m_{p}\left(m_{n}\right)$ is the mass of positive (negative) ion; $T_{p}\left(T_{n}\right)$ is the temperature of positive (negative) ion; $Z_{p}\left(Z_{n}\right) Z_{d}$ is the number of charged particles residing onto the unit grain surface of positive ion (negative ion) dust; e is the magnitude of the charge of an electron; $\mathrm{n}_{\mathrm{p} 0}\left(\mathrm{n}_{\mathrm{n} 0}\right) \mathrm{n}_{\mathrm{d} 0}$ is the positive ion (negative ion) dust number density at equilibrium; $\mathrm{r}$ is the space variable normalized by $\lambda_{\mathrm{Dp}} ; \mathrm{t}$ is the time variable normalized by

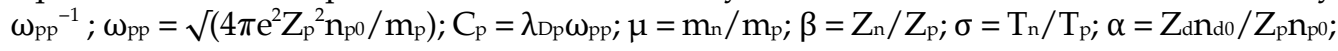
and 1 is $1(-1)$ for negative (positive) dust. 


\section{K-DV SOLITON}

Considering $v=0$ will give us the model equations corresponds to planar (1D) geometry. To obtain the DIA K-dV equation, we introduce the stretched coordinates:

$\zeta=\epsilon^{1 / 2}\left(\mathrm{r}-\mathrm{V}_{\mathrm{p}} \mathrm{t}\right), \tau=\epsilon^{3 / 2} \mathrm{t}$,

where $V_{p}$ is the DIA wave phase speed $(\omega / k)$, and $\epsilon$ is a smallness parameter measuring the weakness of the dispersion $(0<\epsilon<1)$. As we only consider the case, $v=0$ here, $\mathrm{r}$ is representing the space variable in only one dimension. We expand all the dependent variables $\left(\mathrm{n}_{\mathrm{i}}, \mathrm{u}_{\mathrm{i}}\right.$, and $\left.\psi\right)$ in power series of $\epsilon$ to obtain their equilibrium and perturbed parts. Then we have the following set of equations;

$\mathrm{n}_{\mathrm{s}}=1+\epsilon \mathrm{n}^{(1)}+\epsilon^{2} \mathrm{~ns}^{(2)}+\epsilon 3 \mathrm{n}(3) \mathrm{s}+\cdots$,

$\mathrm{u}_{\mathrm{s}}=0+\epsilon \mathrm{u}_{\mathrm{s}}{ }^{(1)}+\epsilon^{2} \mathrm{u}_{\mathrm{s}}{ }^{(2)}+\epsilon^{3} \mathrm{u}_{\mathrm{s}}{ }^{(3)}+\cdots$,

$\psi=0+\epsilon \psi^{(1)}+\epsilon^{2} \psi^{(2)}+\epsilon^{3} \psi^{(3)}+\cdots$,

$\rho=0+\epsilon \rho^{(1)}+\epsilon^{2} \rho^{(2)}+\epsilon^{3} \rho^{(3)}+\cdots$,

where $\epsilon$ is a small parameter measuring the weakness of the dispersion, and $n_{p}{ }^{(1)}, n_{n}{ }^{(1)}, u_{p}$ ${ }^{(1)}, \mathrm{u}_{\mathrm{n}}{ }^{(1)}$, and $\psi^{(1)}$ are the perturbed part of $\mathrm{n}_{\mathrm{p}}, \mathrm{n}_{\mathrm{n}}, \mathrm{u}_{\mathrm{p}}, \mathrm{u}_{\mathrm{n}}$, and $\psi$ respectively. To the lowest order in $\epsilon,(1)-(6)$ give

$\mathrm{u}_{\mathrm{p}}{ }^{(1)}=\left\{\mathrm{V}_{\mathrm{p}} /\left(\mathrm{V}_{\mathrm{p}}{ }^{2}-1\right)\right\} \Psi$,

$\mathrm{n}_{\mathrm{p}}{ }^{(1)}=\left(1 /\left(\mathrm{V}_{\mathrm{p}}{ }^{2}-1\right)\right\} \Psi$,

$\mathrm{un}^{(1)}=\left\{\mathrm{V}_{\mathrm{p}} \beta /\left(\sigma-\mu \mathrm{V}_{\mathrm{p}}{ }^{2}\right)\right\} \Psi$,

$\mathrm{n}_{\mathrm{n}}{ }^{(1)}=\left\{\beta /\left(\sigma-\mu \mathrm{V}_{\mathrm{p}}{ }^{2}\right)\right\} \Psi$,

$\rho^{(1)}=0$,

$\mathrm{V}_{\mathrm{P}}{ }^{2}=(\omega / \mathrm{k})^{2}=(1+\mathrm{C} \sigma) /(1+\mathrm{C} \mu)$,

where $C=1 / \beta(1-1 \alpha)$ and $\Psi=\Psi^{(1)}$. Equation (17) represents the linear dispersion relation for the DIA waves. This clearly indicates that the DIA wave phase speed $\left(\mathrm{V}_{\mathrm{p}}\right)$ depends on only the parameters $1, \alpha, \beta, \sigma$, and $\mu$. To the next higher order of $\epsilon$, we obtain a set of equations, which, after using (12)-(17), can be simplified as

$\mathrm{w} \partial \mathrm{n}_{\mathrm{p}}{ }^{(2)} / \partial \zeta=2 \mathrm{~V}_{\mathrm{p}} \partial \Psi / \partial \tau+\left\{\left(2 \mathrm{~V}_{\mathrm{p}}{ }^{2}+\mathrm{w}\right) / \mathrm{w}^{2}\right\} \Psi \partial \Psi / \partial \zeta+\partial \Psi^{(2)} / \partial \zeta$,

$\partial \mathrm{n}_{\mathrm{n}}{ }^{(2)} / \partial \zeta=-(2 \mu \beta \mathrm{Vp} / \mathrm{z}) \partial \Psi / \partial \tau+\left(\mathrm{k} / \mathrm{z}^{3}\right) \Psi \partial \Psi / \partial \zeta+(\beta / \mathrm{z}) \partial \Psi^{(2)} / \partial \zeta$,

$\partial^{2} \Psi / \partial \zeta^{2}=\left[\mathrm{n}_{\mathrm{p}}^{(2)}-(1-1 \alpha) \mathrm{nn}^{(2)}\right]$.

where $\mathrm{w}=\left(\mathrm{V}_{\mathrm{p}}{ }^{2}-1\right), \mathrm{z}=\left(\sigma-\mu \mathrm{V}_{\mathrm{p}}{ }^{2}\right)$ and $\mathrm{k}=\left(\beta^{2} \mathrm{z}-2 \mu \beta^{2} \mathrm{~V}_{\mathrm{p}}{ }^{2}\right)$. Now combining (18)-(20), we obtain an equation of the form:

$\partial \Psi / \partial \tau+\mathrm{A} \Psi \partial \Psi / \partial \zeta+\mathrm{B} \partial^{3} \Psi / \partial \zeta^{3}=0$,

where

$\mathrm{A}=\left(2 \mathrm{Vp}^{2} / \mathrm{w} 3+1 / \mathrm{w}^{2}-\beta^{2}(1-1 \alpha) / \mathrm{z}^{2}+\mathrm{R}\right) \mathrm{B}$,
$\mathrm{B}=\left(2 \mathrm{~V}_{\mathrm{p}} / \mathrm{w}+2 \mu \beta \mathrm{V}_{\mathrm{p}}(1-\mathrm{l} \alpha) / \mathrm{z}\right)^{-1}$, 
$\mathrm{R}=2 \mu \beta^{2} \mathrm{~V}_{\mathrm{p}}^{2}(1-1 \alpha) / \mathrm{z}^{3}$.

Equation (21) is known as K-dV (Korteweg-de Vries) equation. The stationary localized solution of (21) is given by

$\Psi=\psi \operatorname{msech}^{2}\left[\left(\zeta-\mathrm{U}_{0} \tau\right) / \delta\right]$,

where the amplitude $\psi_{\mathrm{m}}$ and the width $\delta$ are given by $\psi_{\mathrm{m}}=3 \mathrm{U}_{0} / \mathrm{A}$ and $\delta=\sqrt{4 \mathrm{~B}} / \mathrm{U}_{0}$, respectively. $U_{0}$ is the mach number. As $U_{0}>0$, (25) clearly indicates that (i) small amplitude SWs with $\Psi>0$, i.e. positive soliton exists if $\mathrm{A}>0$, (ii) small amplitude SWs with $\Psi<0$, i.e. negative soliton exists if $\mathrm{A}<0$, and (iii) no solitons can exist around $\mathrm{A}=0$.

Figure (1-3) shows the variation of the amplitude of positive (negative) K-dV soliton with $\alpha$ when the system contains negative dust. The amplitude of the positive (negative) K-dV solitons increases (decreases) with $\alpha$. Whereas figures 2 and 4 shows the same variations when the system contains positive dust. For positive dust, the amplitude of the positive (negative) $\mathrm{K}-\mathrm{dV}$ solitons decreases (increases) with $\alpha$. From figure 3, it is clear that no finite amplitude K-dV soliton can exists at $\alpha=1$.

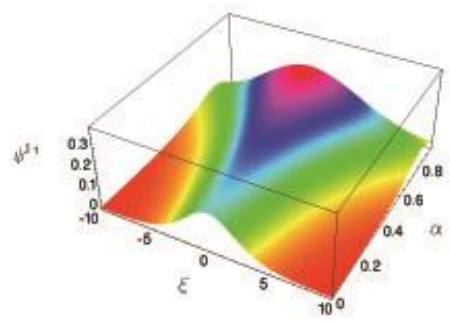

FIG. 1: Showing the variation of the positive K-dV soliton with $\alpha$ at $\mathrm{U}_{0}=0.01, \beta=1, \sigma=1$, and $\mu=0.1$, when the system contains negative dust.

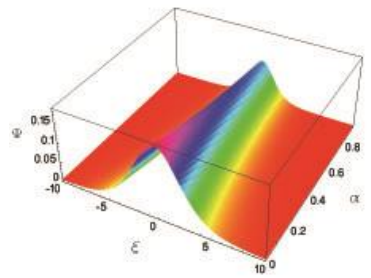

FIG. 2: Showing the variation of the positive K-dV soliton with $\alpha$ at $\mathrm{U}_{0}=0.01, \beta=1, \sigma=1$, and $\mu=0.1$, when the system contains positive dust.

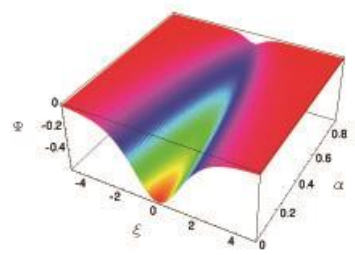

FIG. 3: The variation of the amplitude of negative K-dV soliton with $\alpha$ at $\mathrm{U}_{0}=0.01, \beta=1, \sigma=1$, and $\mu=10$, when the system contains negative dust. 


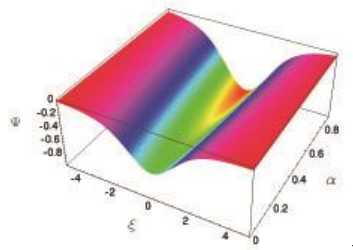

FIG. 4: The variation of the amplitude of negative K-dV soliton with $\alpha$ at $\mathrm{U}_{0}=0.01, \beta=1, \sigma=1$, and $\mu=10$, when the system contains positive dust.

Figures 4 and 5 show the relations between $\psi \mathrm{m}, \beta$, and $\sigma$. The amplitude of positive K-dv soliton increases with $\sigma$, but decreases with $\beta$. Whereas, the amplitude of negative K-dV soliton decreases with $\sigma$, but increases with $\beta$ until $\beta \sim 2.5$.

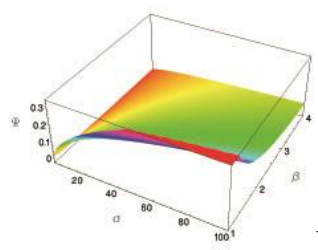

FIG. 5: The variation of the amplitude of $\mathrm{K}-\mathrm{dV}$ soliton with $\beta$ and $\sigma$ at $\mathrm{U}_{0}=0.01, \mu=0.1$.

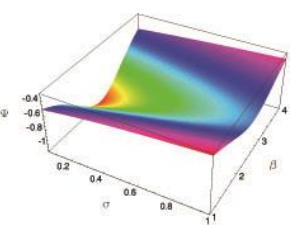

FIG. 6: The variation of the amplitude of negative $\mathrm{K}-\mathrm{dV}$ soliton with $\beta$ and $\sigma$ at $\mathrm{U}_{0}=0.01, \mu$ $=10$.

\section{MK-DV SOLITON}

The K-dV equation is the result of the second order calculation of the $\epsilon$. For plasmas with more than two species, there can arise a situation, where A vanishes at $\mu=\mu_{c}$, and (25) fails to describe nonlinear evolution of perturbation. So, higher order calculation is required at $\mu$ $=\mu \mathrm{c}$. From the third order calculation, which utilizes another set of stretched coordinate, a modified $\mathrm{K}-\mathrm{dV}(\mathrm{mK}-\mathrm{dV})$ equation is obtained to describe the nonlinear evolution near this critical parameter. The stretched coordinates for $\mathrm{mK}-\mathrm{dV}$ equation is

$\zeta=\epsilon\left(r-V_{p} t\right), \tau=\epsilon^{3} t$.

By using (26) in (1)-(6), we find the same values of $\mathrm{n}_{\mathrm{p}}{ }^{(1)}, \mathrm{nn}^{(1)}, \mathrm{u}_{\mathrm{p}}{ }^{(1)}, \mathrm{u}_{\mathrm{n}}{ }^{(1)}$, and $\mathrm{V}_{\mathrm{p}}$, as like as that of K-dV. To the next higher order of $\epsilon$, we obtain a set of equations, which, after using the values of $\mathrm{n}_{\mathrm{p}}{ }^{(1)}, \mathrm{n}_{\mathrm{n}}{ }^{(1)}, \mathrm{u}_{\mathrm{p}}{ }^{(1)}, \mathrm{u}_{\mathrm{n}}{ }^{(1)}$, and $\mathrm{V}_{\mathrm{p}}$, can be simplified as

$\mathrm{n}_{\mathrm{p}}{ }^{(2)}=\left[\mathrm{V}_{\mathrm{p}}{ }^{2} / \mathrm{w}^{3}+1 / 2 \mathrm{w}^{2}\right] \Psi^{2}+[1 / \mathrm{w}] \Psi^{(2)}$,

$\mathrm{n}_{\mathrm{n}}{ }^{(2)}=\left[\beta^{2} / 2 \mathrm{z}^{2}-\mu \beta^{2} \mathrm{~V}_{\mathrm{p}}{ }^{2} / \mathrm{z}^{3}\right] \Psi^{2}+[\beta / \mathrm{z}] \psi^{(2)}$, 
$\mathrm{u}_{\mathrm{p}}^{(2)}=\left[\mathrm{V}_{\mathrm{p}}^{3} / \mathrm{w}^{3}-\mathrm{V}_{\mathrm{p}} / 2 \mathrm{w}^{2}\right] \Psi^{2}+\left[\mathrm{V}_{\mathrm{p}} / \mathrm{w}\right] \Psi^{(2)}$,

$\mathrm{u}_{\mathrm{n}}{ }^{(2)}=-\left[\mathrm{V}_{\mathrm{p}} \beta^{2} / 2 \mathrm{z}^{2}+\mu \beta^{2} \mathrm{~V}_{\mathrm{p}}{ }^{3} / \mathrm{z}^{3}\right] \Psi^{2}+\left[\mathrm{V}_{\mathrm{p}} \beta / \mathrm{z}\right] \Psi(2)$,

$\rho^{(2)}=1 / 2 \mathrm{~A} \Psi^{2}=0$.

where

$\mathrm{A}=\left(2 \mathrm{~V}_{\mathrm{p}}^{2} / \mathrm{w}^{3}+/ \mathrm{w}^{2}-\beta^{2}(1-1 \alpha) / \mathrm{z}^{2}+\mathrm{R}\right)$.

We take the next higher order of $\epsilon$, and get a set of equations:

$\partial \mathrm{n}_{\mathrm{p}}{ }^{(1)} / \partial \tau-\mathrm{V}_{\mathrm{p}} \partial \mathrm{n}_{\mathrm{p}}{ }^{(3)} / \partial \zeta+\partial \mathrm{u}_{\mathrm{p}}{ }^{(3)} / \partial \zeta+\partial / \partial \zeta\left(\mathrm{n}_{\mathrm{p}}{ }^{(1)} \mathrm{u}_{\mathrm{p}}{ }^{(2)}\right)+\partial / \partial \zeta\left(\mathrm{n}_{\mathrm{p}}{ }^{(2)} \mathrm{u}_{\mathrm{p}}{ }^{(1)}\right)=0$,

$\partial \mathrm{n}_{n}{ }^{(1)} / \partial \tau-\mathrm{V}_{\mathrm{p}} \partial \mathrm{n}_{\mathrm{n}}{ }^{(3)} / \partial \zeta+\partial \mathrm{u}_{\mathrm{n}}{ }^{(3)} / \partial \zeta+\partial / \partial \zeta\left(\mathrm{n}_{\mathrm{n}}{ }^{(1)} \mathrm{u}_{\mathrm{n}}{ }^{(2)}\right)+\partial / \partial \zeta\left(\mathrm{n}_{\mathrm{n}}{ }^{(2)} \mathrm{un}^{(1)}\right)=0$,

$\partial \mathrm{u}_{\mathrm{p}}{ }^{(1)} / \partial \tau-\mathrm{V}_{\mathrm{p}} \partial \mathrm{u}_{\mathrm{p}}{ }^{(3)} / \partial \zeta-\mathrm{V}_{\mathrm{p}} \mathrm{n}_{\mathrm{p}}{ }^{(1)} \partial \mathrm{u}_{\mathrm{p}}^{(2)} / \partial \zeta-\mathrm{V}_{\mathrm{p}} \mathrm{n}_{\mathrm{p}}^{(2)} \partial \mathrm{u}_{\mathrm{p}}{ }^{(1)} / \partial \zeta+\partial / \partial \zeta\left(\mathrm{u}_{\mathrm{p}}{ }^{(1)} \mathrm{u}_{\mathrm{p}}{ }^{(2)}\right)+\mathrm{n}_{\mathrm{p}}{ }^{(1)} \mathrm{u}_{\mathrm{p}}{ }^{(1)} \partial \mathrm{u}_{\mathrm{p}}{ }^{(1)} / \partial \zeta$

$+\mathrm{n}_{\mathrm{p}}{ }^{(1)} \partial \psi^{(2)} / \partial \zeta+\mathrm{n}_{\mathrm{p}}{ }^{(2)} \partial \Psi / \partial \zeta+\partial \phi^{(3)} / \partial \zeta+\partial \mathrm{n}_{\mathrm{p}}{ }^{(3)} / \partial \zeta=0$

$\partial \mathrm{u}_{\mathrm{n}}{ }^{(1)} / \partial \tau-\mathrm{V}_{\mathrm{p}} \partial \mathrm{u}_{\mathrm{n}}{ }^{(3)} / \partial \zeta-\mathrm{V}_{\mathrm{p}} \mathrm{n}_{\mathrm{n}}{ }^{(1)} \partial \mathrm{u}_{\mathrm{n}}{ }^{(2)} / \partial \zeta-\mathrm{V}_{\mathrm{p}} \mathrm{n}_{\mathrm{n}}{ }^{(2)} \partial \mathrm{u}_{\mathrm{n}}{ }^{(1)} / \partial \zeta+\mathrm{n}_{\mathrm{n}}{ }^{(1)} \mathrm{u}_{\mathrm{n}}{ }^{(1)} \partial \mathrm{u}_{\mathrm{n}}{ }^{(1)} / \partial \zeta+\partial / \partial \zeta\left(\mathrm{u}_{\mathrm{n}}{ }^{(1)} \mathrm{u}_{\mathrm{n}}{ }^{(2)}\right)+$ $\left.(\sigma / \mu) \partial \mathrm{n}^{(3)} / \partial \zeta-\beta / \mu\left(\mathrm{n}_{\mathrm{n}}{ }^{(1)} \partial \psi^{(2)} / \partial \zeta-\mathrm{n}_{\mathrm{n}}{ }^{(2)} \partial \Psi / \partial \zeta\right)+\beta / \mu \partial \phi^{(3)} / \partial \zeta\right)=0$,

$\partial^{2} \Psi / \partial \zeta^{2}+n_{p}^{(3)}-(1-1 \alpha) n_{n}^{(3)}=0$.

Now, combining (32)-(36), and using the values of $\mathrm{n}_{\mathrm{p}}{ }^{(1)}, \mathrm{n}_{\mathrm{p}}{ }^{(2)}, \mathrm{n}_{\mathrm{n}}{ }^{(1)}, \mathrm{n}_{\mathrm{n}}{ }^{(2)}, \mathrm{u}_{\mathrm{p}}{ }^{(1)}, \mathrm{u}_{\mathrm{p}}{ }^{(2)}, \mathrm{u}_{\mathrm{n}}{ }^{(1)}, \mathrm{u}_{\mathrm{n}}{ }^{(2)}$, and $\rho^{(2)}$ in (32)-(36), we obtain an equation of the form:

$\partial \Psi / \partial \tau+\mathrm{ab} \Psi^{2} \partial \Psi / \partial \zeta+\mathrm{b} \partial^{3} \Psi / \partial \zeta^{3}=0$,

where

$\mathrm{a}=\left\{1 /\left(2 \mathrm{w}^{5}\right)\right\}\left(\mathrm{m}_{1} 15 \mathrm{~V}_{\mathrm{p}}{ }^{4}-\mathrm{m}_{2} 4 \mathrm{~V}_{\mathrm{p}}{ }^{2}+\mathrm{m}_{3}\right)$,

$\mathrm{m}_{1}=1-\left(\beta^{2} \mu^{2} / \mathrm{C}\right)$,

$\mathrm{m}_{2}=1-\left(\beta^{2} \mu^{\sigma} / C\right)$,

$\mathrm{m} 3=1-\left(\beta^{2} \sigma^{2} / C\right)$,

$\mathrm{b}=\left[\mathrm{w}^{2} /\left\{2 \mathrm{~V}_{\mathrm{p}}(1+\mathrm{c} \mu)\right\}\right](1-\mu)^{-32}$.

Equation (37) is known as mK-dV equation. But this equation allows us to study only one type of solitons. In this equation the dispersion term (viz. $\Psi \partial \Psi / \partial \zeta$ ) is absent, but instead of this, there is a dissipation term $(\Psi \partial \Psi / \partial \zeta)$. The stationary localized solution of (37) is, therefore, can be directly given by

$\Psi=\psi \mathrm{msech}[\xi / \Delta]$,

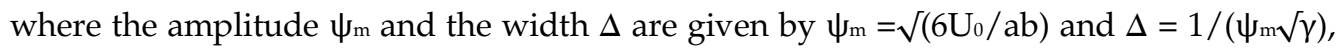
and $\gamma=\mathrm{a} / 6$. 


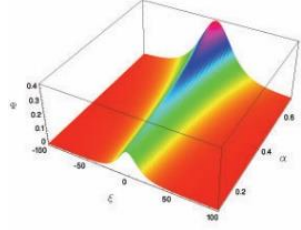

FIG. 7: The variation of the mK-dV soliton with $\alpha$ at $\mathrm{U}_{0}=0.01, \beta=1, \sigma$ $=2$, and $\mu=0.1$ when the system contains negative dust.

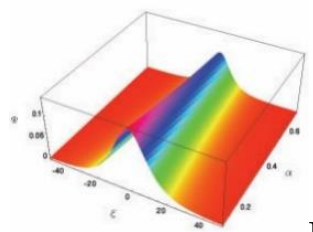

FIG. 8: The variation of the mK-dV soliton with $\alpha$ at $\mathrm{U}_{0}=0.01, \beta=1, \sigma$ $=2$, and $\mu=0.1$ when the system contains positive dust, instead of negative dust.

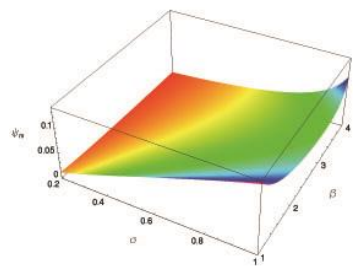

FIG. 9: The variation of the amplitude of $\mathrm{mK}-\mathrm{dV}$ soliton with $\sigma$ and $\beta$ at $\alpha=0.5$ and $\mu=0.1$ when the system contains negative dust.

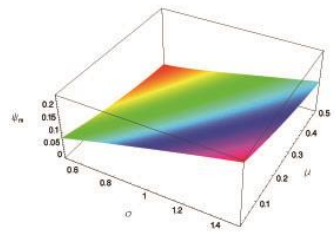

FIG. 10: The variation of the amplitude of $\mathrm{mK}-\mathrm{dV}$ soliton with $\sigma$ and $\mu$ at $\alpha=0.5$ and $\beta=1$ when the system contains negative dust.

Figure 7 shows the variation of the $\mathrm{mK}-\mathrm{dV}$ solitons with $\alpha$ with negative dust and figure 8 shows the variation of the $\mathrm{mK}-\mathrm{dV}$ solitons with $\alpha$ with positive dust. Figure 9 shows the variation of the amplitude of the $\mathrm{mK}-\mathrm{dV}$ soliton with $\sigma$ and $\beta$. Figure 10 shows the variation of the amplitude of the $\mathrm{mK}-\mathrm{dV}$ soliton with $\sigma$ and $\mu$. From figures 7-10, we may conclude:

i. The amplitude and width of $\mathrm{mK}-\mathrm{dV}$ soliton increase with $\alpha$. ii. With the increase of temperature of the negative ion, the amplitude of $\mathrm{mK}-\mathrm{dV}$ soliton also increases. But with the increase of the mass of the negative ion, the amplitude decreases. iii. The amplitude of $\mathrm{mK}-\mathrm{dV}$ soliton first decreases with $\beta$, but after the point $\beta=2.5$, it begins to increase.

\section{GARDNER SOLITON}

It is obvious from (31) that $\mathrm{A}=0$ since $\psi \neq 0$. One can find that $\mathrm{A}=0$ at its critical value $\mu$ $=\mu_{\mathrm{c}}($ which is a solution of $\mathrm{A}=0)$. The solution of $\mathrm{A}=0$ for $\mu$ is gives by

$\mu=\mu_{\mathrm{c}}=\left(2+3 \sigma C-\sigma \beta C^{2}\right) /\left(1-3 \beta C-2 \sigma \beta C^{2}\right)$. 
At $\mu=\mu_{c}, A=0$. So, for $\mu$ around its critical value $\left(\mu_{c}\right)$, i.e. for $\left|\mu-\mu_{c}\right|=\epsilon$ corresponding to $A=A_{0}$, we can express $A_{0}$ as

$\mathrm{A}_{0} \simeq \mathrm{r}(\partial \mathrm{A} / \partial \mu)_{\mu=\mu \mathrm{c}}\left|\mu-\mu_{\mathrm{c}}\right|=\mathrm{s} \epsilon$.

where $\left|\mu-\mu_{\mathrm{c}}\right|$ is a dimension less and small parameter, and can be taken as the expansion parameter $\epsilon$, i.e. $\left|\mu-\mu_{\mathrm{c}}\right| \simeq \epsilon$, and

$\mathrm{s}=6 \mathrm{r} \mathrm{C}_{2} \mathrm{C}_{3} / \mathrm{C}^{2} \mathrm{C}_{1}^{3}+3 \mathrm{r} \beta \mathrm{C}_{2} \mathrm{C}_{3}^{2} / \mathrm{CC}_{1}^{3}+6 \mathrm{r} \beta \mu_{1} \mathrm{C}_{2} \mathrm{C}_{3} / \mathrm{C}_{1}^{3}-3 \mathrm{rC}_{4} \mathrm{C}_{3}^{2} / \mathrm{C}^{2} \mathrm{C}_{1}^{3}+3 \mathrm{rC}_{2} \mathrm{C}_{3}^{2} / \mathrm{C}^{3} \mathrm{C}_{1}^{4}+$ $3 \mathrm{r} \beta \mu_{\mathrm{c}} \mathrm{C}_{2} \mathrm{C}_{3}^{2} / \mathrm{CC}_{1}^{4}-\mathrm{rC}_{4} \mathrm{C}_{3}^{3} / \mathrm{C}^{3} \mathrm{C}_{1}^{4}$,

where $C_{1}=\left(\sigma-\mu_{c}\right), C_{2}=(1+C \sigma), C 3=\left(1+C \mu_{c}\right), C 4=\left(1+C^{2} \beta \sigma\right), r=1$ for $\mu>\mu_{c}$, and $r=-1$ for $\mu<\mu_{\text {c. So, }} \rho^{(2)}$ can be expressed as

$\epsilon^{2} \rho^{(2)} \simeq \epsilon^{3}(1 / 2) s \psi^{2}$,

which, therefore, must be included in the third order Poisson's equation. For the first two lowest orders of $\epsilon$, we get the same set of equations as like as mK-dV (until eq. (31)). To the next higher order of $\epsilon$, we obtain a set of equations:

$\partial \mathrm{n}_{\mathrm{p}}{ }^{(1)} / \partial \tau-\mathrm{V}_{\mathrm{p}} \partial \mathrm{n}_{\mathrm{p}}{ }^{(3)} / \partial \zeta+\partial \mathrm{u}_{\mathrm{p}}^{(3)} / \partial \zeta+\partial / \partial \zeta\left(\mathrm{n}_{\mathrm{p}}^{(1)} \mathrm{u}_{\mathrm{p}}^{(2)}\right)+\partial / \partial \zeta\left(\mathrm{n}_{\mathrm{p}}{ }^{(2)} \mathrm{u}_{\mathrm{p}}^{(1)}\right)=0$,

$\partial \mathrm{n}_{\mathrm{n}}{ }^{(1)} / \partial \tau-\mathrm{V}_{\mathrm{p}} \partial \mathrm{n}_{\mathrm{n}}{ }^{(3)} / \partial \zeta+\partial \mathrm{un}^{(3)} / \partial \zeta+\partial / \partial \zeta\left(\mathrm{n}_{\mathrm{n}}{ }^{(1)} \mathrm{u}_{\mathrm{n}}{ }^{(2)}\right)+\partial / \partial \zeta\left(\mathrm{n}_{\mathrm{n}}{ }^{(2)} \mathrm{u}_{\mathrm{n}}{ }^{(1)}\right)=0$,

$\partial \mathrm{u}_{\mathrm{p}}{ }^{(1)} / \partial \tau-\mathrm{V}_{\mathrm{p}} \partial \mathrm{u}_{\mathrm{p}}{ }^{(3)} / \partial \zeta-\mathrm{V}_{\mathrm{p}} \mathrm{n}_{\mathrm{p}}{ }^{(1)} \partial \mathrm{u}_{\mathrm{p}}{ }^{(2)} / \partial \zeta-\mathrm{V}_{\mathrm{p}} \mathrm{n}_{\mathrm{p}}^{(2)} \partial \mathrm{u}_{\mathrm{p}}{ }^{(1)} / \partial \zeta+\partial / \partial \zeta\left(\mathrm{u}_{\mathrm{p}}{ }^{(1)} \mathrm{u}_{\mathrm{p}}{ }^{(2)}\right)+\mathrm{n}_{\mathrm{p}}{ }^{(1)} \mathrm{u}_{\mathrm{p}}{ }^{(1)} \partial \mathrm{u}_{\mathrm{p}}{ }^{(1)} / \partial \zeta+$ $\mathrm{n}_{\mathrm{p}}{ }^{(1)} \partial \Psi^{(2)} / \partial \zeta+\mathrm{n}_{\mathrm{p}}{ }^{(2)} \partial \Psi / \partial \zeta+\partial \phi^{(3)} / \partial \zeta+\partial \mathrm{n}_{\mathrm{p}}{ }^{(3)} / \partial \zeta=0$,

$\partial \mathrm{un}^{(1)} / \partial \tau-\mathrm{V}_{\mathrm{p}} \partial \mathrm{u}_{\mathrm{n}}{ }^{(3)} / \partial \zeta-\mathrm{V}_{\mathrm{p}} \mathrm{n}_{\mathrm{n}}^{(1)} \partial \mathrm{u}_{\mathrm{n}}{ }^{(2)} / \partial \zeta-\mathrm{V}_{\mathrm{p}} \mathrm{n}_{\mathrm{n}}{ }^{(2)} \partial \mathrm{u}_{\mathrm{n}}{ }^{(1)} / \partial \zeta+\sigma / \mu \partial \mathrm{n}_{\mathrm{n}}{ }^{(3)} / \partial \zeta-\beta / \mu \partial \phi^{(3)} / \partial \zeta+$ $\partial / \partial \zeta\left(u_{n}{ }^{(1)} u_{n}{ }^{(2)}\right)+n_{n}{ }^{(1)} u_{n}{ }^{(1)} \partial u_{n}{ }^{(1)} / \partial \zeta-n_{n}{ }^{(1)}(\beta / \mu) \partial \Psi^{(2)} / \partial \zeta-n_{n}{ }^{(2)}(\beta / \mu) \partial \Psi / \partial \zeta=0$,

$\partial^{2} \Psi / \partial \zeta^{2}+n_{p}{ }^{(3)}-(1-1 \alpha) n_{n}{ }^{(3)}+(s / 2) \Psi^{2}=0$.

Now, combining (48)-(52), we obtain an equation of the form:

$\partial \Psi / \partial \tau+\operatorname{sb} \Psi \partial \Psi / \partial \zeta+\mathrm{ab} \Psi^{2} \partial \Psi / \partial \zeta+\mathrm{b} \partial^{3} \Psi / \partial \zeta^{3}=0$,

where

$\mathrm{a}=\left(1 / 2 \mathrm{w}^{5}\right)\left(\mathrm{m}_{1} 15 \mathrm{~V}_{\mathrm{p}}^{4}-\mathrm{m}_{2} 4 \mathrm{~V}_{\mathrm{p}}{ }^{2}+\mathrm{m}_{3}\right)$,

$\mathrm{m}_{1}=1-\left(\beta^{2} \mu^{2} / \mathrm{C}\right)$,

$\mathrm{m}_{2}=1-\left(\beta^{2} \mu^{\sigma} / \mathrm{C}\right)$,

$\mathrm{m}_{3}=1-\left(\beta^{2} \sigma^{2} / C\right)$,

$\mathrm{b}=\left[\mathrm{w}^{2} /\left\{2 \mathrm{~V}_{\mathrm{p}}(1+\mathrm{c} \mu)\right\}\right](1-\mu)^{-3 / 2}$.

Equation (53) is known as Gardner equation or mixed mK-dV equation. The Gardner equation contains both $\Psi^{2}$ and $\Psi^{3}$ term, i.e., it has both types of solitary wave solution, which

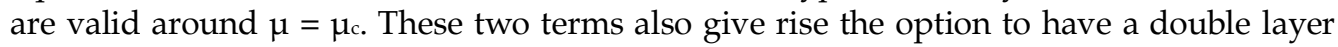
(DLs) wave solution of this equation. But as in this paper we are only interested to analyze 
the SWs solution, we will not look at the DLS solution. The solitary wave solution (Deeba et al., 2012; Deeba and Mamun, 2011) of standard Gardner equation is, therefore, given by

$\Psi=\left[1 / \Psi_{\mathrm{m} 2}-\left(1 / \psi_{\mathrm{m} 2}-1 / \psi_{\mathrm{m} 1}\right) \cosh ^{2}(\xi / \delta)\right]^{-1}$,

where $\gamma=\mathrm{a} / 6$ and $\psi_{\mathrm{m}}=-\mathrm{s} / \mathrm{a}$.

The GSs amplitude $\psi_{\mathrm{m} 1,2}$ and width $\delta$ are given by

$\psi_{\mathrm{m} 1,2}=\psi_{\mathrm{m}}\left[1 \mp \sqrt{(}\left(1+\mathrm{U}_{0} / \mathrm{V}_{0}\right)\right]$,

where

$\mathrm{V}_{0}=\mathrm{s}^{2} \mathrm{~b} / 6 \mathrm{a}$,

$\mathrm{U}_{0}=(\mathrm{sb} / 3) \psi_{\mathrm{m} 1,2}+(\mathrm{ab} / 6) \psi_{2} \mathrm{~m} 1,2$,

$\left.\delta=2 / \sqrt{(}-\gamma \psi_{\mathrm{m} 1} \psi_{\mathrm{m} 2}\right)=\sqrt{ }\left(\mathrm{b} / \mathrm{U}_{0}\right)$.

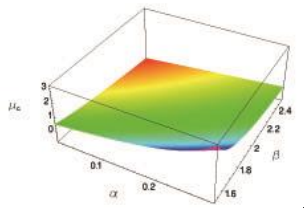

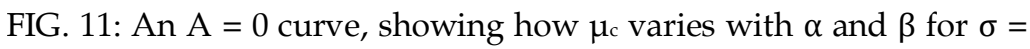
0.9 in a multi-ion plasma system consisting negatively charged dust.

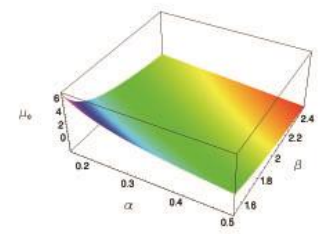

FIG. 12: An $A=0$ curve, showing how $\mu_{\mathrm{c}}$ varies with $\alpha$ and $\beta$ for $\sigma=$ 1.5 in a multi-ion plasma system consisting positively charged dust.

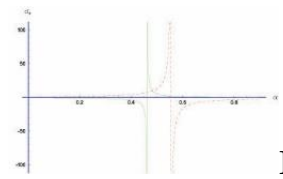

FIG. 13: The variation of $\sigma_{c}$ with $\alpha$ for $\beta=0.1$ and $\mu=0.2$. The green line corresponds to a multi-ion plasma system consisting negatively charged dust and the red dotted line corresponds to a multi-ion plasma system consisting positively charged dust.

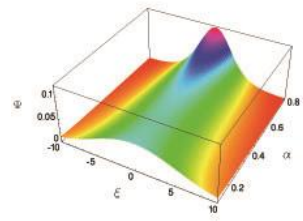

FIG. 14: The variation of amplitude of positive GSs with $\alpha$ at $\beta=1, \sigma=$ 10 , and $\mu=1$ in a multi-ion plasma system consisting negatively charged dust. 


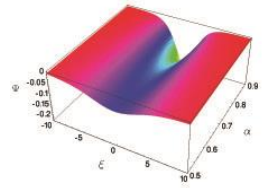

FIG. 15: The variation of amplitude of negative GSs with $\alpha$ at $\beta=1, \sigma=10$, and $\mu=1$ in a multi-ion plasma system consisting negatively charged dust.

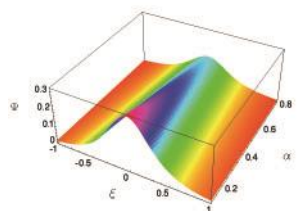

FIG. 16: The variation of amplitude of positive GSs with $\alpha$ at $\beta=1, \sigma=$ 10 , and $\mu=4$, in a system consisting positive dust.

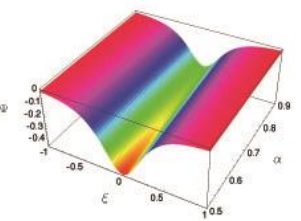

FIG. 17: The variation of amplitude of negative GSs with $\alpha$ at $\beta=1, \sigma=$ 1 , and $\mu=4$, in a system consisting positive dust.

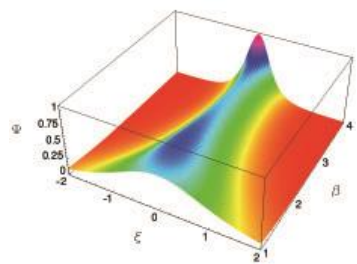

FIG. 18: The variation of the amplitude of positive GSs with $\beta$ at $\alpha=$ 0.5 at $\mathrm{U}_{0}=0.01, \sigma=10$, and $\mu=1$ when the system contains negative dust.

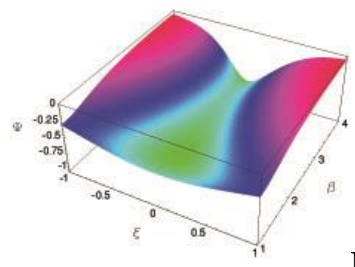

FIG. 19: The variation of the amplitude of negative GSs with $\beta$ at $\alpha=$ $0.5, \mathrm{U}_{0}=0.01, \sigma=10$, and $\mu=1$. 


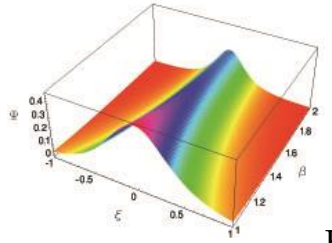

FIG. 20: The variation of the amplitude of positive GSs with $\beta$ at $\alpha=$ $0.5, \mathrm{U}_{0}=0.01, \sigma=1$, and $\mu=0.1$, in a system consisting positive dust.

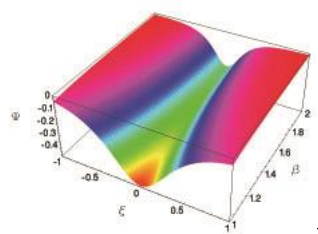

FIG. 21: The variation of the amplitude of negative GSs with $\beta$ at $\alpha=$ $0.5, \mathrm{U}_{0}=0.01, \sigma=1$, and $\mu=0.1$, in a system consisting positive dust.

Equation (59) represents the SWs solution of Gardner equation. The SWs profile is positive if $\mathrm{A}>0$ and negative if $\mathrm{A}<0$. Therefore, it can be concluded that positive (negative) SWs exist at $\mu>\mu_{\mathrm{c}}\left(\mu<\mu_{\mathrm{c}}\right)$ i.e., $\mathrm{j}=1(\mathrm{j}=-1)$. To find the parametric regimes for which the positive and negative SWs exist, we have analytically analyzed $A$, and obtain $A\left(\mu=\mu_{c}\right)=0$ surface plots for both negative and positive dust. The $A\left(\mu=\mu_{c}\right)=0$ surface plot for negative (positive) dust is shown in fig. 11 (fig. 12). These mean that the positive (negative) SWs exist for a set of dusty plasma parameters corresponding to any point which is much above (below) the $A\left(\mu=\mu_{c}\right)=0$ surfaces shown in figures 11 and 12. In one word, the SWs can exist either above or below of the surface curve, but no SWs will be found at the surface of the $\mathrm{A}=0$. Because this is the limit $(\mathrm{A}=0)$ for which $\mathrm{K}-\mathrm{dV}$ types equation breaks down. Our success of this model is to analysis the waves around the vicinity of this limit. $A=0$ can be also solved in terms of $\sigma=\sigma_{\text {c. }}$.

Fig. 13 shows the variation of $\sigma_{c}$ with different parameter. Figure (14-15) shows the variation of the positive (negative) Gardner solitons with $\alpha$ for negative dust. And figure (18-19) shows the variation of the GSs with $\beta$. From figures (14-19), it has been found that, the magnitude of the amplitude of GSs increases with both $\alpha$ and $\beta$. Same figures for a system consisting positive dust, have been drawn in figures (16-21). It has been found that, the amplitude of GSs decreases with both $\alpha$ and $\beta$ for positive dust.

\section{NONPLANAR SOLITON}

Now, if we proceed with $v \neq 0$, we will be able to analyze the nonplanar GSs. $v=1$ represents a nonplanar cylindrical geometry, whereas, $v=2$ represents a nonplanar spherical geometry. To do so, we will introduce the same stretched coordinates as like as mK-dV:

$\zeta=\epsilon(\mathrm{r}-\mathrm{Vpt}), \tau=\epsilon 3 \mathrm{t}$,

where $\epsilon$ has usual meaning as mentioned before, and $V_{p}$ (normalized by $C_{i}$ ) is the phase speed of the perturbation mode. Following the same procedure and set of equations of expansion in power series of $\epsilon$ like before, and substitute into the resulting equations [(1)(6) expressed in terms of $\zeta$ and $\tau]$, we get, different sets of equations in various powers of $\epsilon$. To the lowest order in $\epsilon$, we obtain the values of $u_{p}{ }^{(1)}, n_{p}{ }^{(1)}, u_{n}(1), n_{n}{ }^{(1)}, \rho^{(1)}$, and $V_{P}{ }^{2}$ as that 
of $\mathrm{k}-\mathrm{dV}$ equation. The expression for $\mathrm{V}_{\mathrm{P}}^{2}$ represents the linear dispersion relation for the DIA waves propagating in a dusty plasma under consideration. This equation clearly indicates that the DIA wave phase speed $\left(\mathrm{V}_{\mathrm{p}}\right)$ depends not only on 1 , but also on $\alpha, \beta, \sigma$, and $\mu$. To the next higher order in $\epsilon$, we obtain a set of equations, same as that of $\mathrm{mK}-\mathrm{dV}$ equation, (28-32). It is obvious from (32) that $A=0$ since $\psi \neq 0$. To the next higher order of $\epsilon$, using the solution of $A=0$ at its critical value $\mu=\mu_{c}$, i.e. eq. (46) and eq. 48 , we obtain a set of equations:

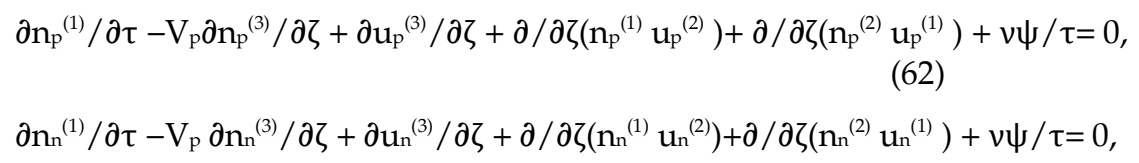

$\partial \mathrm{u}_{\mathrm{p}}{ }^{(1)} / \partial \tau-\mathrm{V}_{\mathrm{p}} \partial \mathrm{u}_{\mathrm{p}}{ }^{(3)} / \partial \zeta-\mathrm{V}_{\mathrm{p}} \mathrm{n}_{\mathrm{p}}{ }^{(1)} \partial \mathrm{u}_{\mathrm{p}}{ }^{(2)} / \partial \zeta-\mathrm{V}_{\mathrm{p}} \mathrm{n}_{\mathrm{p}}{ }^{(2)} \partial \mathrm{u}_{\mathrm{p}}{ }^{(1)} / \partial \zeta+\partial / \partial \zeta\left(\mathrm{u}_{\mathrm{p}}{ }^{(1)} \mathrm{u}_{\mathrm{p}}{ }^{(2)}\right)+\mathrm{n}_{\mathrm{p}}{ }^{(1)} \mathrm{u}_{\mathrm{p}}{ }^{(1)}$ $\partial \mathrm{u}_{\mathrm{p}}{ }^{(1)} / \partial \zeta+\mathrm{n}_{\mathrm{p}}{ }^{(1)} \partial \Psi^{(2)} / \partial \zeta+\mathrm{n}_{\mathrm{p}}{ }^{(2)} \partial \Psi / \partial \zeta+\partial \phi^{(3)} / \partial \zeta+\partial \mathrm{n}_{\mathrm{p}}{ }^{(3)} / \partial \zeta=0$,

$\partial \mathrm{u}_{\mathrm{n}}{ }^{(1)} / \partial \tau-\mathrm{V}_{\mathrm{p}} \partial \mathrm{u}_{\mathrm{n}}{ }^{(3)} / \partial \zeta-\mathrm{V}_{\mathrm{p}} \mathrm{n}_{\mathrm{n}}{ }^{(1)} \partial \mathrm{u}_{\mathrm{n}}{ }^{(2)} / \partial \zeta-\mathrm{V}_{\mathrm{p}} \mathrm{n}_{\mathrm{n}}{ }^{(2)} \partial \mathrm{u}_{\mathrm{n}}{ }^{(1)} / \partial \zeta+\sigma / \mu \partial \mathrm{n}_{\mathrm{n}}{ }^{(3)} / \partial \zeta-\beta / \mu \partial \phi^{(3)} / \partial \zeta+$ $\partial / \partial \zeta\left(u_{n}{ }^{(1)} u_{n}{ }^{(2)}\right)+n_{n}{ }^{(1)} u_{n}{ }^{(1)} \partial u_{n}{ }^{(1)} / \partial \zeta-n_{n}{ }^{(1)}(\beta / \mu) \partial \psi^{(2)} / \partial \zeta-n_{n}{ }^{(2)}(\beta / \mu) \partial \Psi / \partial \zeta=0$,

$\partial^{2} \Psi / \partial \zeta^{2}+n_{p}{ }^{(3)}-(1-1 \alpha) n_{n}{ }^{(3)}+(s / 2) \Psi^{2}=0$.

Now, combining (62)-(66), we obtain an equation of the form:

$\partial \Psi / \partial \tau+(\nu / 2 \tau) \psi+\operatorname{sb} \Psi \partial \Psi / \partial \zeta+a b \Psi^{2} \partial \Psi / \partial \zeta+b \partial^{3} \Psi / \partial \zeta^{3}=0$,

where

$\mathrm{a}=\left(1 / 2 \mathrm{w}^{5}\right)\left(\mathrm{A}^{(1)} 15 \mathrm{Vp} 4-\mathrm{A}^{(2)} 4 \mathrm{~V}_{\mathrm{p}}^{2}+\mathrm{A}^{(3)}\right)$,

$\mathrm{b}=\left[\mathrm{w}^{2} /\left\{2 \mathrm{~V}_{\mathrm{p}}(1+\mathrm{c} \mu)\right\}\right](1-\mu)^{-3 / 2}$.

with

$\mathrm{A}^{(1)}=1-\left(\beta^{2} \mu^{2} / \mathrm{C}\right)$,

$\mathrm{A}^{(2)}=1-\left(\beta^{2} \mu^{\sigma} / C\right)$,

$\mathrm{A}^{(3)}=1-\left(\beta^{2} \sigma^{2} / C\right)$,

Equation (67) is the modified Gardner ( $\mathrm{mG}$ ) equation. The term 'modified' has been used because of the extra term $(v / 2 \tau) \psi$, which arises as a result of the effects of the nonplanar geometry. $v=1(2)$, corresponds to a cylindrical (spherical) geometry, while $v=0$ corresponds to a 1D as well as planar geometry. As Eq. (67) contains both $\psi^{2}$ and $\psi^{3}$ term, it must support both SW and DL solutions. An exact analytic solution of (67) is not possible.

Therefore, we have numerically solved (67), and have studied the effects of cylindrical and spherical geometries on time-dependent DIA-GSs. The initial condition that we have used in our numerical analysis, is in the form of the SWs solution of $(67)$ without the term $(v / 2 \tau)$ $\phi$. 


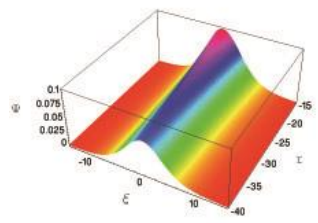

FIG. 22: The effects of the cylindrical geometry on the compressive DIA-GSs for $\alpha=0.5, \beta=1, \sigma=10, \mu=1$, and $\mathrm{U}_{0}=0.1$, when the system contains negative dust.

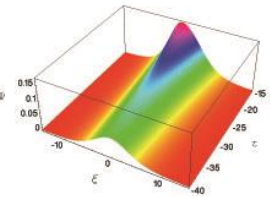

FIG. 23: The effects of the spherical geometry on the compressive DIAGSs for $\alpha=0.5, \beta=1, \sigma=10, \mu=1$, and $U_{0}=0.1$, when the system contains negative dust.

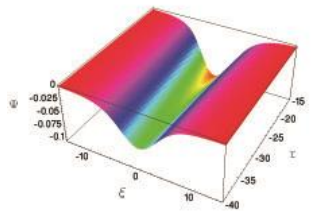

FIG. 24: The effects of the cylindrical geometry on the negative DIAGSs for $\alpha=0.5, \beta=1, \sigma=10, \mu=1$, and $U_{0}=0.1$, when the system contains negative dust.

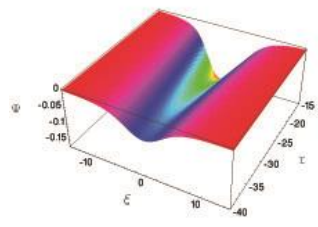

FIG. 25: The effects of the spherical geometry on the negative DIA-GSs for $\alpha=0.5, \beta=1, \sigma=10, \mu=1$, and $U_{0}=0.1$, when the system contains negative dust.

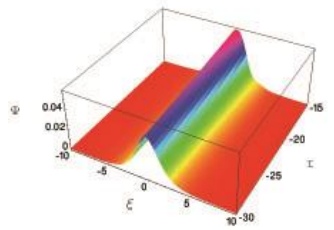

FIG. 26: The effects of the cylindrical geometry on the compressive DIA-GSs for $\alpha=0.5, \beta=1, \sigma=1, \mu=100$, and $U_{0}=0.1$, when the system contains positive dust. 


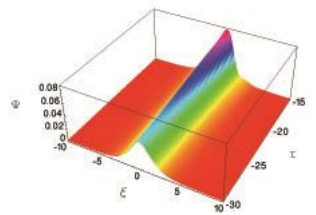

FIG. 27: The effects of the spherical geometry on the compressive DIAGSs $\alpha=0.5, \alpha=0.5, \beta=1, \sigma=1, \mu=100$, and $U_{0}=0.1$, when the system contains positive dust.

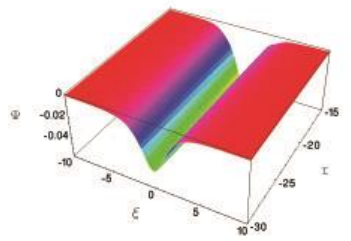

FIG. 28: The effects of the cylindrical geometry on the negative DIAGSs for $\alpha=0.5, \alpha=0.5, \beta=1, \sigma=1, \mu=100$, and $U_{0}=0.1$, when the system contains positive dust.

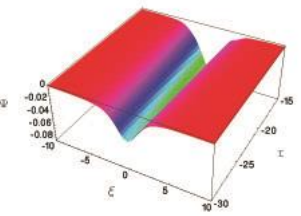

FIG. 29: The effects of the spherical geometry on the negative DIA-GSs for $\alpha=0.5, \alpha=0.5, \beta=1, \sigma=1, \mu=100$, and $U_{0}=0.1$, when the system contains positive dust.

Figures 22 and 24 show how the effects of the cylindrical geometry, when the system contains negative dust, modify the DIA-GSs, while figure 25 shows how the effects of the spherical geometry modify the DIA-GSs, when the system contains negative dust.

Figures 28 -29 show how the effects of the cylindrical and spherical geometry on the negative DIA-GSs respectively, for positive dust.

Again, figures 26-27 show how the effects of the cylindrical and spherical geometry on the compressive DIA-GSs respectively, when the system contains positive dust.

The numerical solutions of (67) reveal that for a large value of $\tau$ (e.g. $\tau=-40)$, the spherical and cylindrical solitary waves are similar to 1D planar structures. This is because for a large value of $\tau$ the term $(v / 2 \tau) \psi$, which is due to the effects of the cylindrical or spherical geometry, is no longer dominant. However, as the value of $\tau$ decreases, the term $(v / 2 \tau) \psi$ becomes dominant, and the spherical and cylindrical DIA-GSs differ from the 1D planar ones. It is found that as the value of $\tau$ decreases, the amplitude of these localized pulses increases. It is also found that the amplitude of the cylindrical DIA-GSs is larger than those of 1D planar ones, but smaller than that of the spherical ones.

\section{ANALYSIS AND Discussion}

In this section, an in-depth investigation has been made on several types of DIA solitons (K$\mathrm{dV}, \mathrm{mK}-\mathrm{dV}$, and Gardner) and corresponding nonplanar SWs in a multi-ion dusty plasma system (consisting of positive and negative ions, and arbitrary charged stationary dust). A 
comparative discussion among these three types of solutions has been also presented. It has been found that, the K-dV solitons and mK-dV solitons are not valid for $\mu=\mu_{c}$, which vanishes the nonlinear coefficients of the K-dV equation. The observations, which have been obtained from this investigation can be summarized as follows:

1. The multi-ion dusty plasma system under consideration supports finite amplitude GSs (Gardner Solitons), whose basic features (polarity, amplitude, width, etc.) depend on the ion and dust number densities, temperature, masses, and charges of ions.

2. The K-dV solitons, which is governed by the K-dV equation, do not exists around $\mu=\mu_{\text {c. }}$ The amplitude of positive (negative) K-dV solitons increases (decreases) with the increase of concentration of negative dust.

3. The existence of only positive mK-dV solitons have been observed. Unlike positive K-dV solitons, its amplitude also increases with the increase of concentration of negative dust.

4. If the polarity of dust particles has been altered, above properties of $\mathrm{K}-\mathrm{dV}$ and mk-dV solitons will also alter.

5. With the increase of the temperature of negative ion, the amplitudes of both $\mathrm{K}-\mathrm{dV}$ and $\mathrm{mK}-\mathrm{dV}$ soli tons increase. But the amplitudes of both $\mathrm{K}-\mathrm{dV}$ and $\mathrm{mK}-\mathrm{dV}$ solitons first decreases with the increase of the charge of negative ion, but after a certain point it begins to increase.

6. GSs which are governed by Gardner equation, are found to exist around $\mu=\mu_{\mathrm{c}}$, and are shown be different from K-dV and $\mathrm{mK}-\mathrm{dV}$ solitons.

7. At $\mu<\mu_{\mathrm{c}}$, positive GSs exist, whereas at $\mu>\mu_{\mathrm{c}}$, negative GSs exist.

8. The magnitude of the amplitudes of positive and negative GSs increase with both $\alpha$ and $\beta$, when the system contains negative dust, but decreases when the system contains positive dust.

9. No GSs can exists at $\mu \leq \sigma$.

10. The properties of the nonplanar (cylindrical and spherical) DIA-GSs are significantly different from those of the 1D planar ones.

11. The amplitude of the cylindrical DIA-GSs is larger than those of planar ones, but smaller than that of the spherical ones. 12. For a large value of $\tau$ (e.g. $\tau=-40)$, the spherical and cylindrical solitary waves are similar to 1D planar structures.

\section{CONCLUSION}

We finally propose to carry out a laboratory experiment that may be able to test the theory that we presented in our present work.

\section{ACKNOWLEDGEMENT}

Research help of the authority of Jahangirnagar University, Savar, Dhaka University of Engineering \& Technology, Gazipur and Kazi Agro Limited, Dhaka, is gratefully acknowledged. 


\section{REFERENCES}

Akhtar T., Hossain M. M. and Mamun A. A., (2013a), Chinese Physical Society and IOP Publishing Ltd, 59, 6.

Akhtar T., Hossain M. M. and Mamun A. A., (2013b), Astrophysics and Space Science, 344, 105-112.

Alinejad H. and Mamun A. A. (2010), Phys. Plasmas 17, 123704.

Asaduzzaman M., Mamun A. A., (2013), Astrophysics and SpaceScience, 341,535-542.

Barkan A., Merlino R. L., and D’Angelo N., (1995), Phys. Plasmas 2, 3563.

Bharuthram R. and Shukla P. K., (1992), Planet. Space Sci. 40, 973.

Charles C, (2007), Plasma Sources Sci. Technol. 16, R1R25.

Cooney I. L., Gavin M. T., Tao I., and Lonngren K. E., (1991), IEEE Trans. Plasma Sci. 19, 1259.

Das G. C. and Tagare S. G., (1975), Plasma Phys. 17, 1025.

Deeba F. and Mamun A., (2011), Open Journal of Acoustics 3, 70-75.

Deeba F., Tasnim S., and Mamun A. A., (2012), IEEE Trans. Plasma Sci. 40, 2247.

Duha S. S. and Mamun A. A., (2009), Phys. Lett A. 373, 1287.

Duha S. S., (2009), Phys. Plasmas 16, 113701.

Fortov V. E., Ivlev A. V., Khrapak S. A., Khrapak A. G., and Morfill G. E., (2005), Phys. Rep. 421, 1.

Ghosh S. and Bharuthram R., (2008), Astrophys. Space Sci. 314, 121.

Homann A., Melzer A., Peters S., and Piel A., (1997), Phys. Rev. E 56, 7138.

Ishihara O., (2007), J. Phys. D 40, R121.

Jacquinot J., McVey B. D., and Scharer J. E., (1977), Phys. Rev. Lett. 39, 88.

Kim S. H. and Merlino R. L., (2006), Phys. Plasmas 13, 052118.

Kim S. H. and Merlino R. L., (2007), Phys. Rev. E 76, 035401.

Klumov B. A., Ivlev A. V., and Morfill G., (2003), JETP Lett. 78, 300.

Lonngren K. E., (1983), Plasma Phys. 25, 943.

Ludwig G. O., Ferreira J. L., and Nakamura Y., (1984), Phys. Rev. Lett. 52, 275.

Luo Q. Z., Angelo N. D', and Merlino R. L., (1999), Phys. Plasmas 7, 3457.

Luo Q. Z., DAngelo N., and Merlino R. L., (1998), Phys. Plasmas 5, 2868.

Mamun A. A. and Deeba F., (2012), Plasma Phys. Rep. 38, 1

Mamun A. A. and Shukla P. K., (2002), IEEE Trans. Plasma Sci. 30, 720.

Mamun A. A. and Shukla P. K., (2002), Phys. Plasmas 9, 1468.

Mamun A. A. and Shukla P. K., (2003), Phys. Plasmas 10, 1518.

Mamun A. A. and Shukla P. K., (2009), Europhysics Letters, 87, 2.

Mamun A. A. and Shukla P. K., (2009a), Phys. Rev. E 80, 037401.

Mamun A. A. and Shukla P. K., (2009b), Europhys. Lett. 87, 25001.

Mamun A. A., Cairns R.A., and Shukla P. K., (2009), Phys. Lett. A 373, 2355.

Mamun A. A., Shukla P. K., and Eliasson B., (2009), Phys. Plasmas 16, 114503. 
Mamun A. A., Shukla P. K., and Eliasson B., (2009), Phys. Rev. E 80, 046406.

Mendis D. A. and Rosenberg M., (1994), Annu. Rev. Astron. Astrophys. 32, 419.

Merlino R. L. and Kim S. H., (2006), Appl. Phys. Lett. 89, 091501.

Merlino R. L., Barkan A., Thompson C., and D'Angelo N., (1998), Phys. Plasmas 5, 1607.

Morfill G. E. and Ivlev A. V., (2009), Rev. Mod. Phys. 81, 1353.

Morfill G. E., Tsytovich V. N., and Thomas H., (2003), Plasma Phys. Rep. $29,1$.

Nakamura Y. and Sharma A., (2001), Phys. Plasmas 8, 3921.

Nakamura Y. and Tsukabayashi I., (1984), Phys. Rev. Lett. 52, 2356.

Nakamura Y., (1982), IEEE Trans. Plasma Sci. 10, 180.

Nakamura Y., Bailung H., and Shukla P. K., (1999), Phys. Rev. Lett. 83, 1602.

Nakamura Y., Odagiri T., and Tsukabayashi I., (1997), Plasma Phys. Controlled Fusion 39, 105.

Popel S. I. and Yu M. Y., (1995), Contrib. Plasma Phys. 35, 103.

Popel S. I., Golub A. P., and Losseva T. V., (2001), JETP Lett. 74, 396.

Popel S. I., Golub A. P., Losseva T. V., Ivlev A. V., Khrapak S. A., and Morfill G. E., (2003), Phys. Rev. E 67, 056402.

Roychoudhury R. and Chatterjee P., (1999), Phys. Plasmas 6, 406.

Sauer K., Bogdanvo A., and Baumgrtel K., (1994), Geophys. Res. Lett. 21, 2255.

Sauer K., Dubinin E., and McKenzie J. F., (2003), Nonlinear Processes Geophys. 10, 121.

Sauer K., Dubinin E., Baumgrtel K., and Bogdanvo A., (1996), Geophys. Res. Lett. 23, 3643.

Sayed F., Haider M. M., Mamun A. A., Shukla P. K., Eliasson B., and Adhikary N., (2008), Phys. Plasmas 15, 063701.

Shukla P. K. and Eliasson B., (2009), Rev. Mod. Phys. 81, 25.

Shukla P. K. and Mamun A. A., (2003), New J. Phys. 5, 17.

Shukla P. K., (2000), Phys. Plasmas 7, 1044.

Shukla P. K., (2001), Phys. Plasmas 8, 1791.

Tajiri M. and Tuda M., (1985), J. Phys. Soc. Jpn. 54, 19.

Tsytovich V. N., Morfill G. E., and Thomas H., (2002), Plasma Phys. Rep. 28, 623.

Verheest F., Hellberg M. A., and Kourakis I., (2008), Phys. Plasmas 15, 112309.

Washimi H. and Taniuti T., (1966), Phys. Rev. Lett. 17, 996.

Watanabe N., Watari T., Hiroe S., Hidekuma S., Kumazawa R., Adati K., and Sato T., (1978), Plasma Phys. 20, 333.

Watanabe S., (1984), J. Phys. Soc. Jpn. 53, 950.

Weingarten A., Arad R., Maron Y., and Fruchtman A., (2001), Phys. Rev. Lett. 87, 115004. 